\title{
B Semileptonic Decays at High Recoil Momentum
}

\section{T. H. Davies, E. Follana and K. Y. Wong*}

Department of Physics and Astronomy, University of Glasgow, Glasgow, G12 8QQ, UK

\section{G. P. Lepage}

Laboratory of Elementary Particle Physics, Cornell University, Ithaca, New York 14853, USA

\section{J. Shigemitsu}

Department of Physics, The Ohio State University, Columbus, Ohio 43210, USA

\begin{abstract}
We explore the possibility of studying $B \rightarrow \pi l v$ semileptonic decays at large recoil momentum. Our methods include the use of a random-wall source for the pion to reduce statistical errors, and different smearing functions are used for the B meson to improve the overlap with the ground state. We observe, in general, a factor of 3-4 improvement in the signal-to-noise ratio in correlation functions if random-wall propagators are used.
\end{abstract}

The XXV International Symposium on Lattice Field Theory

July 30 - August 42007

Regensburg, Germany

\footnotetext{
*Speaker.
} 


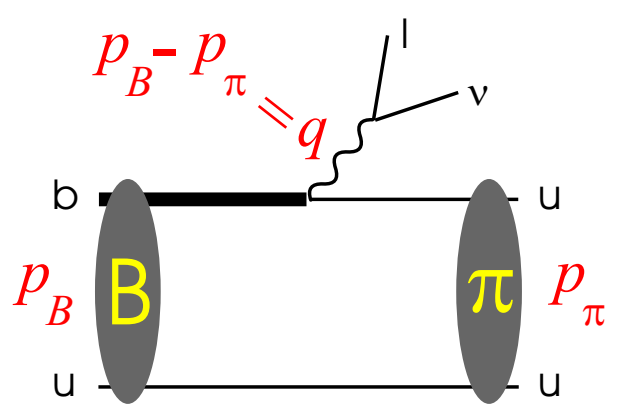

Figure 1: Kinematics of $B \rightarrow \pi l v$ semileptonic decay. $p_{B}$ is the momentum of the $B$ meson and $p_{\pi}$ is the momentum of the pion, $q$ is the momentum transfer.

\section{Introduction}

Precise determination of the form factors $f_{0}\left(q^{2}\right), f_{+}\left(q^{2}\right)$ in $B \rightarrow \pi l v$ semileptonic decays is crucial to the determination of the CKM-matrix element $\left|V_{u b}\right|$. Fig. 1 shows the kinematics of the process. Lattice QCD provides a first principles nonperturbative approach to calculate the from factors in semileptonic decays. Standard simulation methods, however, are problematic in the low $q^{2}$ region when the pion has large recoil momentum. Simulation results are limited to $q^{2} \gtrsim 15 \mathrm{GeV}^{2}$ [1], 2] while experimental data spans the entire $q^{2}$ range. Large recoil momenta are difficult for lattice calculations because statistical errors, which are set by $E(p)-E(p=0)$, become worse when the hadrons have large momenta. In addition, discretization errors, which are set by $a^{2} p^{2}$, increase as the pion momentum increases.

In order to utilize all the experimental data and thereby reduce the experimental error on $\left|V_{u b}\right|$, it is important to develop new simulation techniques to cover the low $q^{2}$ region. One method is to reduce the pion momentum by using a lattice frame in which the $\mathrm{B}$ meson is moving in the opposite direction to the pion; to describe a b quark with large velocity on the lattice the "moving-NRQCD" formalism is used [3, 4, 5, 6]. In this work we concentrate on reducing the statistical errors with the use of a random-wall source for the light quark propagator. In particular we explore the possibility of simulating at $a p_{\pi}=\frac{2 \pi}{L}(3,0,0)$, which corresponds to $q^{2} \sim 10 \mathrm{GeV}^{2}$ in the B meson rest frame. We test this on MILC coarse lattices where discretization errors are about $8 \%$ at this momentum; errors will be much smaller on the fine (3\%) and super-fine (1.5\%) lattices. We also use the Highly Improved Staggered Quark (HISQ) action [7, 8] for the valence light quarks.

\section{Pion 2-Point Function}

A zero-momentum random-wall source is generated by setting the color vector on each site of a time slice ${ }^{1}$ to a three-component random complex unit vector $\vec{\eta}(x)$ [9]. This random source is used in matrix inversion to obtain the staggered-quark propagator $g(y, x)$

$$
\tilde{g}(y) \equiv \sum_{x} g(y, x) \vec{\eta}(x)=\sum_{x} M_{y, x}^{-1} \vec{\eta}(x)
$$

\footnotetext{
${ }^{1}$ In practice we also put the source on a random time slice.
} 
where $M_{y, x}$ is the kernel of the staggered-quark action. It is more convenient to work in the naïvequark basis. The 4-component naïve-quark propagator $S(y, x)$ is given by [8]

$$
S(y, x) \equiv\left\langle\psi^{l}(y) \bar{\psi}^{l}(x)\right\rangle=g(y, x) \Omega(y) \Omega^{\dagger}(x),
$$

where

$$
\Omega(x)=\prod_{\mu=0}^{3}\left(\gamma_{\mu}\right)^{x_{\mu}} .
$$

The pion correlator in the naïve-quark basis becomes

$$
\begin{aligned}
\left\langle J_{5}(y) J_{5}(x)\right\rangle & =\left\langle\left(\bar{\psi}^{l}(y) \gamma_{5} \psi^{l}(y)\right)\left(\bar{\psi}^{l}(x) \gamma_{5} \psi^{l}(x)\right)\right\rangle \\
& =\operatorname{Tr}\left[\gamma_{5} \psi^{l}(y) \bar{\psi}^{l}(x) \gamma_{5} \psi^{l}(x) \bar{\psi}^{l}(y)\right] \\
& =\operatorname{Tr}\left[\gamma_{5} S(y, x) \gamma_{5} \gamma_{5} S^{\dagger}(y, x) \gamma_{5}\right] \\
& =\operatorname{Tr}\left[\Omega(y) \Omega^{\dagger}(x) \Omega(x) \Omega^{\dagger}(y)\right] \operatorname{tr}\left[|g(y, x)|^{2}\right] \\
& =4 \operatorname{tr}\left[|g(y, x)|^{2}\right]
\end{aligned}
$$

in terms of the staggered-quark propagator. Here $\operatorname{Tr}[\ldots]$ is a trace over spinor indices while $\operatorname{tr}[\ldots]$ is taken over the color indices. Therefore to construct the pion correlation function at zero momentum we take the magnitude square of $\tilde{g}(y)$ at the sink and sum over spatial sites, and divide by the number of sites $N$

$$
\frac{1}{N} \sum_{y} \tilde{g}^{*}(y) \tilde{g}(y)=\frac{1}{N} \sum_{y, x, x^{\prime}} g^{*}\left(y, x^{\prime}\right) g(y, x) \vec{\eta}^{*}\left(x^{\prime}\right) \vec{\eta}(x) .
$$

Since the averaged correlator has contributions only from where the quark and antiquark start at the same spatial site, i.e., $x=x^{\prime}$, the random-wall source simulates many-point source, and thereby increases the statistics.

For correlator at finite momentum $k$, an additional phase is added to the source

$$
\tilde{g}^{ \pm}(y) \equiv \sum_{x} g(y, x) e^{ \pm i \frac{k}{2} x} \vec{\eta}(x)=\sum_{x} M_{y, x}^{-1} e^{ \pm i \frac{k}{2} x} \vec{\eta}(x)
$$

In this case we multiple $\tilde{g}^{+}(y)$ and $\left(\tilde{g}^{-}(y)\right)^{*}$ with an explicit insertion of $e^{i k x}$ at the sink and sum over spatial sites.

Fig. 2 compares the pion 2-point functions obtained with a local and a random source at $a p_{\pi}=(0,0,0)$ and $a p_{\pi}=\frac{2 \pi}{L}(3,0,0)$ on MILC coarse lattices. The advantage of using a randomwall source is clearly demonstrated, with statistical errors about 5 times smaller compared to the local case. To fit the 2-point functions we employ a Bayesian technique [10] and do a multipleexponential fit

$$
C^{(2)}(t)=\sum_{k=0}^{N_{\pi}-1}(-1)^{k t} a_{k} e^{-E_{\pi}^{k} t}
$$

We use 5 exponentials $N_{\pi}=5$ (i.e., 3 normal states and 2 oscillating states). We observe a factor of 2-3 improvement in the ground state energy and the amplitude for the random-wall source. 

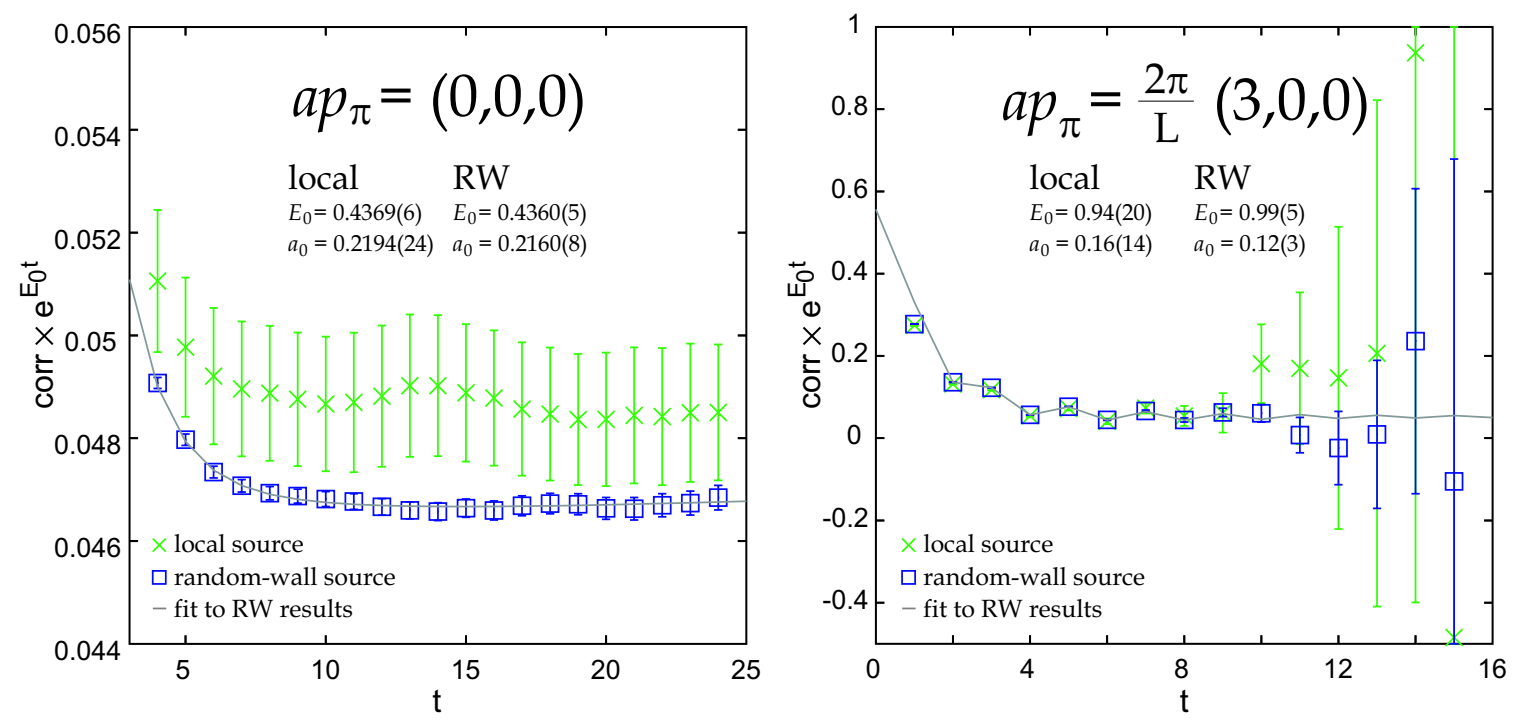

Figure 2: Pion 2-point functions obtained with a local source and a random source on MILC coarse lattices $\left[a m_{\text {sea }}=.01 / .05, a m_{\text {val }}=.05465\left(\sim m_{s}\right)\right.$ for $a p_{\pi}=(0,0,0) ; a m_{\text {sea }}=.02 / .05, a m_{\text {val }}=.02675\left(\sim m_{s} / 2\right)$ for $\left.a p_{\pi}=\frac{2 \pi}{L}(3,0,0)\right]$. Fit results for ground state energy $E_{0}$ and amplitude $a_{0}$ are also shown.

\section{Heavy-Light 2-Point Function}

The heavy-light correlator is

$$
\begin{aligned}
\left\langle J_{5}(y) J_{5}(x)\right\rangle & =\left\langle\left(\bar{\psi}^{l}(y) \gamma_{5} \psi^{Q}(y)\right)\left(\bar{\psi}^{Q}(x) \gamma_{5} \psi^{l}(x)\right)\right\rangle \\
& =\operatorname{Tr}\left[\gamma_{5} \psi^{l}(x) \bar{\psi}^{l}(y) \gamma_{5} \psi^{Q}(y) \bar{\psi}^{Q}(x)\right] \\
& =\operatorname{Tr}\left[\gamma_{5} \gamma_{5} S^{\dagger}(y, x) \gamma_{5} \gamma_{5} G(y, x)\right] \\
& =\operatorname{Tr}\left[\left(\Omega^{\dagger}(y) g^{*}(y, x)\right) G(y, x) \Omega(x)\right] .
\end{aligned}
$$

Here $G(y, x)$ is the heavy-quark propagator. We use NRQCD for the b quark

$$
G(x, t+1)=\left(1-\frac{\delta H}{2}\right)\left(1-\frac{H_{0}}{2 n}\right)^{n} U_{t}^{\dagger}(x)\left(1-\frac{H_{0}}{2 n}\right)^{n}\left(1-\frac{\delta H}{2}\right) G(x, t),
$$

and $H=H_{0}+\delta_{H}$ is the $O(1 / M)$ improved lattice NRQCD Hamiltonian [11, 12]. In order to combine with the light-quark propagator, the heavy-quark propagator must be initialized with the same random noise $\vec{\eta}(x)$

$$
G(x, t=0)=\sum_{x^{\prime}} \Omega\left(x^{\prime}\right) \Phi\left(\left|x^{\prime}-x\right|\right) \vec{\eta}\left(x^{\prime}\right),
$$

where $\Phi\left(\left|x^{\prime}-x\right|\right)$ is the smearing function centered at $x^{\prime}$. Since the smearing function depends on the magnitude of $x^{\prime}-x$ only, $G(x, t=0)$ can be computed efficiently by applying translation to $\Phi(|x|)$, the smearing function with center located at the origin, when doing the summation. At the sink we multiply the heavy-quark propagator with $\Omega^{\dagger}(y) \tilde{g}^{*}(y) \times \Phi(|y|)$ and sum over spatial sites and divide by $N$. Again only the terms with matching random numbers survive after the ensemble 

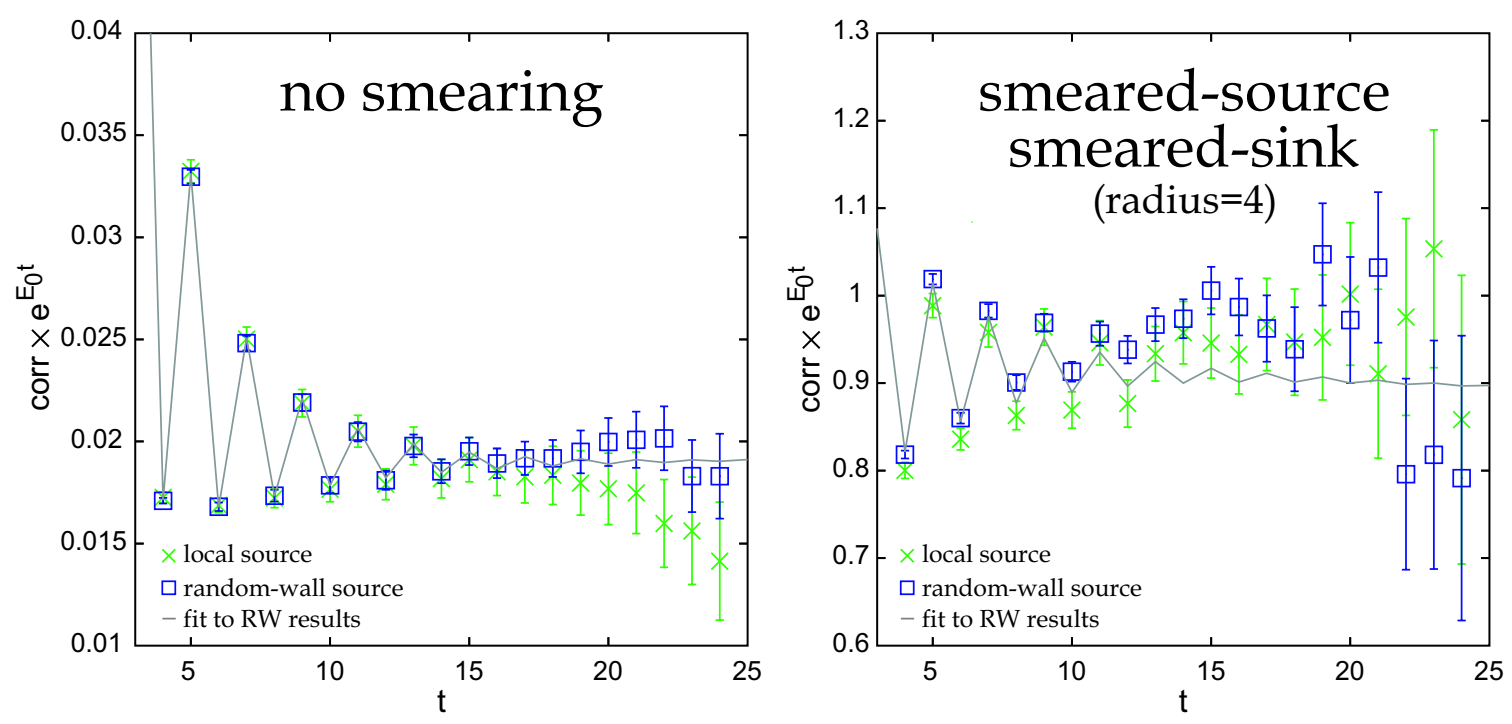

Figure 3: Heavy-light 2pt-functions on MILC coarse lattices $\left[a_{\text {sea }}=.01 / .05, a m_{\text {val }}=.05465\left(\sim m_{s}\right)\right.$, $\left.a m_{b}=2.8\right]$. Gaussian smearing with radius $a r=4$ is used and results are shown for local-source, local-sink [LL] and smeared-source, smeared-sink [SS]. A matrix fit of LL, LS, SL, SS gives [local] $E_{0}=0.5532(15)$, $a_{0}=0.1402(10)$, and $[\mathrm{RW}] E_{0}=0.5559(7), a_{0}=0.1424(5)$.

average, and the correlation function is the average of all contributions from where the heavy quark and light quark start at the same site.

Fig. 3 shows the results obtained with a local source and a random source, with and without smearing. The random-wall results are again more accurate, although the improvement is not as significant as for the light-light correlators. Gaussian smearing is used and results are shown for local-source, local-sink [LL] (i.e., no smearing) and smeared-source, smeared-sink [SS]. The plots show that the advantage of using random-wall propagators decreases if smearing is used. It is therefore crucial to fit the local and smeared results together. We fit LL, LS, SL, SS simultaneously in a matrix fit using 5 exponentials ( 3 normal states and 2 oscillating states) and find that a smearing function of radius $a r=4$ gives the least relative errors for the ground state energy and amplitude.

\section{3-Point Function}

We are interested in the 3-point function

$$
\begin{aligned}
\left\langle J_{5}(y) V_{\mu}(z) J_{5}(x)\right\rangle & =\left\langle\left(\bar{\psi}^{l}(y) \gamma_{5} \psi^{l}(y)\right)\left(\bar{\psi}^{l}(z) \gamma_{\mu} \psi^{Q}(z)\right)\left(\bar{\psi}^{Q}(x) \gamma_{5} \psi^{l}(x)\right)\right\rangle \\
& =\operatorname{Tr}\left[\gamma_{5} \psi^{l}(y) \bar{\psi}^{l}(z) \gamma_{\mu} \psi^{Q}(z) \bar{\psi}^{Q}(x) \gamma_{5} \psi^{l}(x) \bar{\psi}^{l}(y)\right] \\
& =\operatorname{Tr}\left[\gamma_{5} \gamma_{5} S^{\dagger}(z, y) \gamma_{5} \gamma_{\mu} G(z, x) \gamma_{5} S(x, y)\right] \\
& =\operatorname{Tr}\left[\left(\Omega^{\dagger}(z) g^{*}(z, y)\right) \gamma_{5} \gamma_{\mu} G(z, x) \gamma_{5}(\Omega(x) g(x, y))\right] .
\end{aligned}
$$

with $x_{0}<z_{0}<y_{0}$. To compute the current we initialize $G(z, x)$ with $\gamma_{5} \times \Omega(x) \tilde{g}(x)$ and then propagate the heavy quark backward ${ }^{2}$ in time to the current insertion point $z$, where it turns into a

\footnotetext{
${ }^{2}$ Backward means to the opposite direction of the light-quark propagator.
} 

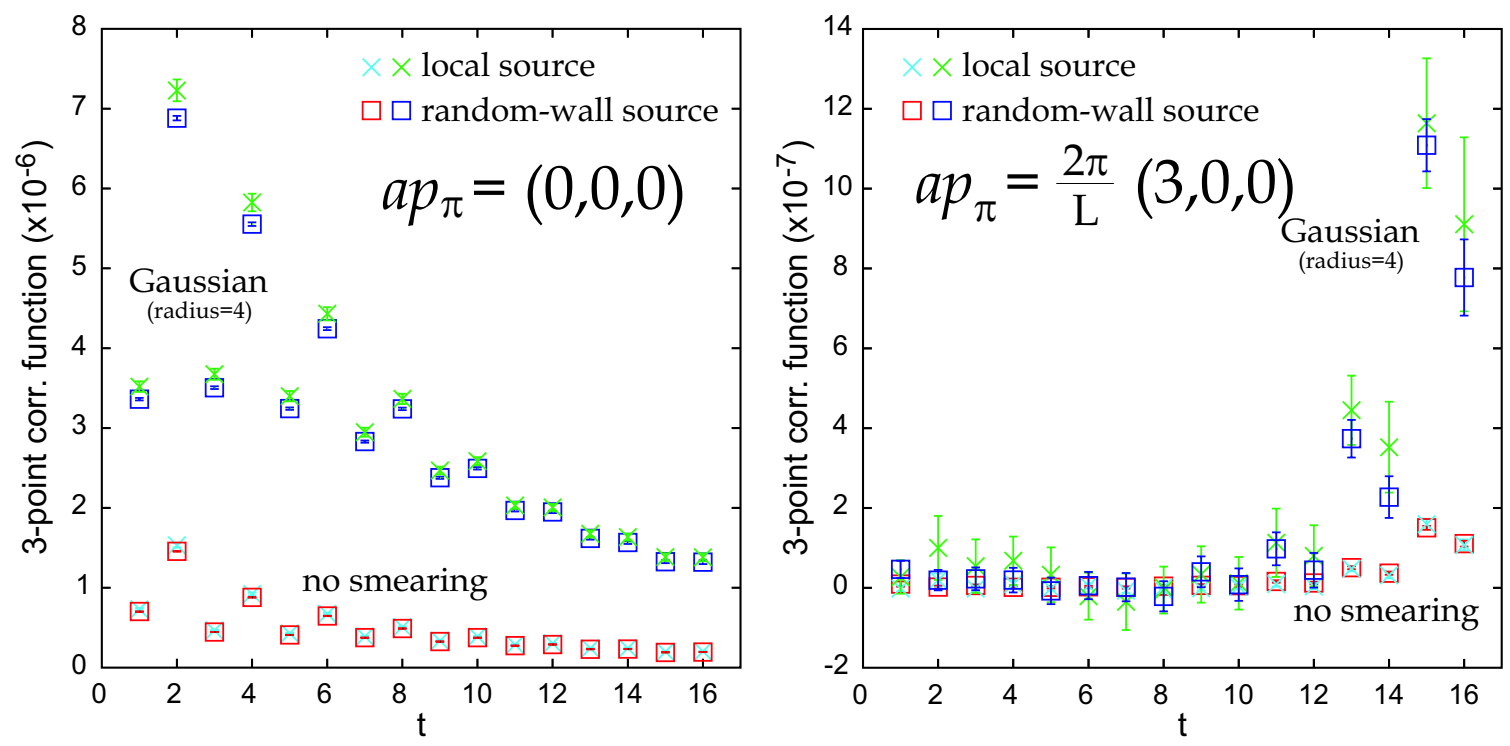

Figure 4: FIG. 5. The 3-point functions (corresponding to the temporal vector currents) obtained with local and random-wall propagators on MILC coarse lattices [the same ensembles as in Fig. 2]. The B meson starts at $t=0$ and the pion is at $t=16$. The random-wall results are 3-4 more accurate compared to the results obtained using local propagators. In particular we obtain, for no smearing, $v_{00}=0.0605(39)$ [local], $v_{00}=0.0597(7)\left[\mathrm{RW}\right.$ ] for $a p_{\pi}=(0,0,0)$, and $v_{00}=0.029(49)$ [local], $v_{00}=0.043(33)$ [RW] for $a p_{\pi}=\frac{2 \pi}{L}(3,0,0)$.

light quark; more precisely, we take the trace of the product of $G(z, x)$ and $\Omega^{\dagger}(z) \tilde{g}^{*}(z) \times \gamma_{5} \gamma_{\mu}$ at the insertion point $z$. This setup is not merely convenient and efficient, but the same computer code can be used for local and random-wall propagators ${ }^{3}$. We only have to divide the random-wall results by an extra factor of $N$ since there are $N$ times more contributions like the one in Eq. (4.1), each from a different source point $x$, to the averaged correlator.

In Fig. 4 we plot the temporal vector currents calculated with local and random-wall propagators for pion momenta $a p_{\pi}=(0,0,0)$ and $a p_{\pi}=\frac{2 \pi}{L}(3,0,0)$. Results again clearly show that statistical noises can be suppressed substantially by the use of random sources. The 3-point function has the functional form

$$
C^{(3)}(t)=\sum_{k=0}^{N_{\pi}-1} \sum_{l=0}^{N_{B}-1}(-1)^{k t}(-1)^{l(T-t)} a_{k} a_{l} v_{k l} e^{-E_{\pi}^{k} t} e^{-E_{B}^{l}(T-t)}
$$

where $T=y_{0}-x_{0}$. To extract the matrix element $v_{00}$ we fit the 3-point function, the 2-point function (Fig. 2) and the heavy-light 2-point function (Fig. 3) simultaneously using $N_{\pi}=N_{B}=5$. Fit results are given along with the graphs. We find that random-wall results are about 5 times more accurate compared to local case at $a p_{\pi}=(0,0,0)$, and about a factor of 2 better at $a p_{\pi}=\frac{2 \pi}{L}(3,0,0)$.

\footnotetext{
${ }^{3}$ In the local case we read in local propagators $\tilde{g}(x)=\sum_{y^{\prime}} g\left(x, y^{\prime}\right) \delta_{y y^{\prime}}=g(x, y)$ and $\tilde{g}(z)=g(z, y)$; in the random-wall case we read in the random-source propagators Eq. (2.1).
} 


\section{Conclusion}

The major problem in studying semileptonic decays on the lattice is the exponential growth of statistical errors in correlation functions as the pion momentum increases. In this work we explored the possibility of reducing statistical noises using random-wall light-quark propagators. We demonstrated that correlation functions obtained with random sources have much better signal-tonoise ratios, with statistical errors about 3-4 times smaller than those obtained with local propagators. One should therefore improve the current lattice simulations at small pion momenta using random-wall propagators. Encouraging results were also obtained at pion momentum as large as $a p_{\pi}=\frac{2 \pi}{L}(3,0,0)$ (corresponding to $q^{2} \sim 10 \mathrm{GeV}^{2}$ ), although statistical errors are still too large for the results to be useful. Work is in progress, e.g. fitting correlation functions with different $T$ simultaneously, to further improve the calculations.

\section{Acknowledgments}

This work was supported by PPARC (UK) and the DOE and NSF (USA). We thank the MILC Collaboration for making their unquenched gauge configurations available. The computations were done on computer clusters at Fermilab and QCDOCX.

\section{References}

[1] M. Okamoto et al., Nucl. Phys. Proc. Suppl. 140:461-463 (2005) [hep-lat/0409116].

[2] E. Dalgic et al., Phys. Rev. D73:074502 (2006) [hep-lat/0601021].

[3] S. Hashimoto and H. Matsufuru, Phys. Rev. D54:4578 (1996) [hep-lat/9511027].

[4] J. H. Sloan, Nucl. Phys. Proc. Suppl. 63, 365 (1998) [hep-lat/9710061].

[5] K. M. Foley and G. P. Lepage, Nucl. Phys. Proc. Suppl. 119, 635 (2002) [hep-lat/0209135].

[6] A. Dougall et al., PoS LAT2005, 219 (2006) [hep-lat/0509108].

[7] E. Follana et al., Nucl. Phys. Proc. Suppl. 129, 447 (2004) [arXiv:hep-lat/0311004].

[8] E. Follana et al., Phys. Rev. D75:054502 (2007) [arXiv:hep-lat/0610092].

[9] C. Aubin et al., MILC Collaboration, Phys. Rev. D70:114501 (2004) [hep-lat/0407028].

[10] G. P. Lepage et al., Nucl. Phys. Proc. Suppl. 106, 12 (2002) [hep-lat/0110175].

[11] G. P. Lepage et al., Phys. Rev. D46:4052 (1992) [arXiv:hep-lat/9205007].

[12] A. Gray et al., Phys. Rev. D72:094507 (2005) [arXiv:hep-lat/0507013]. 